\title{
Analytical parametric analysis of the contact problem of human buttocks and negative Poisson's ratio foam cushions
}

\author{
Yun-Che Wang ${ }^{a}$, Roderic Lakes ${ }^{\mathrm{a}, \mathrm{b}, \mathrm{c}, *}$ \\ a Department of Engineering Physics, Engineering Mechanics Program, University of Wisconsin-Madison, \\ 147 Engineering Research Building, 1500 Engineering Drive, Madison, WI 53706-1687, USA \\ $\mathrm{b}$ Department of Biomedical Engineering, Materials Science Program, University of Wisconsin-Madison, \\ 147 Engineering Research Building, 1500 Engineering Drive, Madison, WI 53706-1687, USA \\ ${ }^{c}$ Rheology Research Center, University of Wisconsin-Madison, 147 Engineering Research Building, \\ 1500 Engineering Drive, Madison, WI 53706-1687, USA
}

Received 28 July 2001; received in revised form 9 May 2002

\begin{abstract}
Analytical investigations on the contact problems between two homogeneous and isotropic soft bodies were performed to simulate the contact of human buttocks and seat cushions. The cushion materials' Poisson's ratio were allowed to be negative. The human buttocks were modeled as an ideal sphere with radius $15 \mathrm{~cm}$, and assumed to have a low Young's modulus and a Poisson's ratio close to 0.5. These parameters were held constant during our analysis. Peak contact pressure was reduced by adjusting the contour curvature of cushions according to Hertz theory, as expected. Moreover, analysis by both the Hertz model and a finite thickness 3D elasticity model showed that using negative Poisson's ratio cushions could further reduce the pressure. Negative Poisson's ratio cushions may be beneficial in the prevention of pressure sores or ulcers in the sick and in reduction of pressure-induced discomfort in seated people. (c) 2002 Elsevier Science Ltd. All rights reserved.
\end{abstract}

Keywords: Poisson's ratio; Polymer foam; Hertz theory; Asymptotic solution

\section{Introduction}

Pressure ulcers are a significant health care problem. In nursing homes $7-23 \%$ of the patients have the problem (Smith, 1995). In a study of the positions of pressure sores, the most common sites for them are the sacrum or coccyx for $36 \%$, the buttocks over the ischial tuberosities for $15 \%$, heels for $12 \%$, ankles for $7 \%$, and 13\% for other sites (Brienza et al., 1996). The major explanation for the occurrence of pressure ulcers at the site of the ischial tuberosities is the loading condition that caused tissue damage. One may conclude that

\footnotetext{
${ }^{*}$ Corresponding author. Address: Department of Engineering Physics, Materials Science Program, University of WisconsinMadison, 147 Engineering Research Building, 1500 Engineering Drive, Madison, WI 53706-1687, USA. Tel.: +1-608-265-8697; fax: +1-608-263-7451.

E-mail address: lakes@engr.wisc.edu (R. Lakes).
} 
the ulcers can be potentially preventable by providing an improved weight distribution on the buttocks while sitting, even for ulcers at the sacrum and coccyx (Dabnichki et al., 1994). To that end, superior seat cushions may be of service in reducing peak pressures. Such cushions may also be more comfortable in general use. The feeling of discomfort is subjective, and the quantification of pressure-induced discomfort is a psychophysical problem. Nevertheless many procedures for rating the discomfort, based on contact pressure measurements, were developed and conducted clinically for seating comfort evaluation (Shen and Parsons, 1997). In addition to the traditional development of wheelchair cushions (Brienza et al., 1999), negative Poisson's ratio foams are considered here since they give rise to new degrees of freedom which may aid design of better cushions which reduce peak contact pressure.

The creation of negative Poisson's ratio foams (Lakes, 1987) is based on changing the shape of foam cell structures to be re-entrant, or bulging inward. Such foams can exhibit negative Poisson's ratio as small as -0.7 for polymer foams, and -0.8 for metal foams. For potential applications, the design considerations (Lakes, 1993) on stress concentrations, Saint Venant's effects (Lakes, 1992), and stress distributions and curvatures in bending problems with negative Poisson's ratio materials have been clearly pointed out. As for seat cushions, the fabrication process has been scaled up to cushion size by Loureiro and Lakes (1997), Lowe and Lakes (2000) experimentally concluded that the contact pressure distribution decreases as sample density decreases for both conventional and re-entrant foams. No substantial influence of the cushion thickness has been observed. In addition, the negative Poisson's ratio foams showed a reasonably linear relationship between stress and strain up to more than $40 \%$ compressive strain, compared to $5 \%$ strain of the conventional foams (Lakes, 1987; Choi and Lakes, 1992). Therefore, the densification of foam due to bottoming or long term creep can be largely reduced, and then the incremental stiffness during the use period will be much more controllable than with traditional foam cushions. However, the theoretical analysis of the influence of negative Poisson's ratios on mechanical contact problems, as reviewed below, still seems to be beyond the research works available so far.

As for the theoretical or numerical analysis of the contact problems, Johnson (1985) summarizes the classical methods, known as Hertz theory, in which a frictionless interface condition is assumed, for two 3D ellipsoidal deformable bodies in contact. Under the same assumptions and following the 3D elasticity equations, Vorovich and Ustinov (1959) used the Fredholm integral equation approach to solve the contact problem between a rigid sphere and a half-space elastic layer with finite thickness. However, due to the complexity of the equations, only asymptotic solutions were obtained. Keer (1964) extended the results from Vorovich and Ustinov to the contact problem of an elastic sphere and a finite thickness elastic layer. In this formulation, for thick-layer problems, the valid solutions can be calculated iteratively, but for thinlayer ones, they only can be solved numerically. In the 3D linear elasticity context, Sakamoto et al. (1996) proposed a closed-form series solution for the contact problem with a finite thickness elastic layer, and Yoffe (1984) extended the Hertz theory for wider contact problems. However, both of them are only suitable for rigid indenters. In addition to the analytical approach, several numerical methods were proposed by different authors. Paul and Hashemi (1980) solved the displacement compatibility equation iteratively to obtain contact pressure, Kalker and van Ranfen (1972) minimized the total elastic strain energy with the constraints of no interference outside the contact region and positive pressure inside, and Singh and Paul (1974) solved the governing integral equation numerically. By using the finite element method, Dabnichki et al. (1994) analyzed the deformation and stress distribution of a supported buttock in a relatively realistic material model. Todd and Thacker (1994) used the finite element method to build a threedimensional computer model of the human buttocks, and did a numerical simulation of in vivo conditions. Kosior et al. (1999) used a numerical method, combining boundary element and domain decomposition methods, to study the bilateral and unilateral contact problems with or without considering friction effects.

The objective of the present analysis is to investigate the effect of the full range of Poisson's ratios, including negative values, on the peak pressure between two contacting elastic bodies and on their deformation. To simplify and illustrate the physical problem, we follow the Hertzian contact assumptions. 
The contact problem is first analyzed via Hertz contact theory. Then we adopt 3D linear elasticity to study the contact problem between an elastic sphere and a finite-thickness elastic layer. The analysis allows the geometrical nonlinearity of contact but assumes material linearity. The nonlinearity of foam and tissue at sufficiently large strain is acknowledged. The fact that the buttock is not a perfect sphere is also acknowledged. The rationale for the present approach is that it allows an analytical procedure, hence clear interpretation of parametric studies. The analysis is intended for application to seat cushions, however an extended range of Poisson's ratio may have broader implications in contact problems.

\section{Hertz analysis model}

Based on the Hertzian assumptions and the analogy with potential theory on the uniformly charged ellipsoid problem, the pressure distribution $P(x, y)$ on an elastic ellipsoidal indenter under loading, force $F$ normal to the contact tangent plane, in the contact region, is (Johnson, 1985)

$$
\begin{aligned}
& P_{z}(x, y)=P_{\max } \sqrt{1-\frac{x^{2}}{a^{2}}-\frac{y^{2}}{b^{2}}}, \quad \text { and } \quad P_{\max }=\frac{3}{2} \frac{F}{\pi a b}, \\
& \delta=\frac{P D}{\pi} \int_{0}^{\infty} \frac{\mathrm{d} \xi}{\sqrt{\left(a^{2}+\xi\right)\left(b^{2}+\xi\right) \xi}}
\end{aligned}
$$

where

$$
\begin{array}{ll}
D=\frac{3}{4}\left(\frac{1-v_{1}^{2}}{E_{1}}+\frac{1-v_{2}^{2}}{E_{2}}\right), & \text { or } \\
D=\frac{3}{8}\left(\frac{1-v_{1}}{G_{1}}+\frac{1-v_{2}}{G_{2}}\right) . &
\end{array}
$$

The two sets of material constants $E_{1}, v_{1}$, and $E_{2}, v_{2}$ represent Young's modulus and Poisson's ratio for the indenter and cushion separately. $\delta$ is the maximum distance of relative approach between the two bodies, sometimes is called motion between the two bodies. The origin of the coordinates is at the center of the elliptic contact region, and $x$ - and $y$-axes coincide with the major and minor axes of the ellipse within the contact surface. $Z$-axis is the direction normal to the contacting tangent plane, as shown in Fig. 1, for the undeformed configuration.

The unknown variables $a$ and $b$ are the length of the major and minor axes of the elliptic contact region, which can be calculated by solving the following nonlinear algebraic equations simultaneously (Johnson, 1985).

$$
\begin{aligned}
& A=\frac{F D}{\pi} \operatorname{Lim}_{c \rightarrow 0}\left[\int_{0}^{\infty} \frac{\mathrm{d} \xi}{\left(a^{2}+\xi\right) \sqrt{\left(a^{2}+\xi\right)\left(b^{2}+\xi\right)\left(c^{2}+\xi\right)}}\right], \\
& B=\frac{F D}{\pi} \operatorname{Lim}_{c \rightarrow 0}\left[\int_{0}^{\infty} \frac{\mathrm{d} \xi}{\left(b^{2}+\xi\right) \sqrt{\left(a^{2}+\xi\right)\left(b^{2}+\xi\right)\left(c^{2}+\xi\right)}}\right],
\end{aligned}
$$

For the general shape of the contacting ellipsoidal bodies, the constants $A$ and $B$ depend on the principal radii $R_{i}$ and $R_{j}$ of curvature of the two objects in contact, as presented by Fessler and Ollerton (1957). If the shapes of the two contacting objects are spherical with radius $R$ and $R^{\prime}$, the parameters in the above equations can be analytically integrated and simplified as follows. 


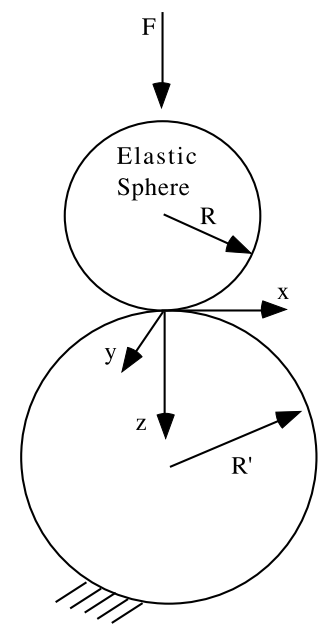

Fig. 1. Schematic diagram for Hertz contact model.

$$
\begin{aligned}
& A=B=\frac{1}{2}\left(R+R^{\prime}\right), \quad a=b=F^{1 / 3}\left[\frac{D R R^{\prime}}{R+R^{\prime}}\right]^{1 / 3}, \\
& P_{\max }=\frac{3}{2} \frac{F}{\pi a b}, \quad \delta=F^{2 / 3}\left[D^{2}\left(\frac{1}{R}+\frac{1}{R^{\prime}}\right)\right]^{1 / 3},
\end{aligned}
$$

where for the contact problem of an elastic sphere with radius $R$ and a half-plane cushion, whose radius $\left(R^{\prime}\right)$ approaches infinity, the maximum pressure along the $z$ direction, $P_{\max }$, and the maximum relative motion, $\delta_{\max }$ are

$$
\begin{aligned}
& P_{\max }=\frac{3}{2 \pi} F^{1 / 3} D^{-2 / 3} R^{-2 / 3}, \\
& \delta_{\max }=F^{2 / 3} D^{2 / 3} R^{-1 / 3} .
\end{aligned}
$$

\section{3D elasticity with finite thickness model}

Actual cushions have a finite thickness. To obtain a more realistic model of the pressure distribution, finite thickness models are considered, as shown in Fig. 2.

After applying the Love's strain function to the Navier's equation in a 3D elasticity framework for axisymmetric problems, we can obtain the mathematical model, which is a single biharmonic PDE with mixed boundary values, for the physical problem. By performing a Hankel transformation, in particular, Keer (1964) shows the indentation of a layer of normalized thickness $h=H / a$, in which the physical thickness, $H$, is normalized to the radius $(a)$ of the contact area, by an elastic sphere can be solved by the following two integral equations. The flat surface area of the cushion is assumed to be infinite.

$$
\int_{0}^{\infty}\left\{\left[\frac{\cosh (2 s h)-1}{2 \operatorname{sh}+\sinh (2 s h)}\right] c_{1}+c_{2}\right\} Q(s) J_{0}(s \rho) \mathrm{d} s=\delta-\varphi(\rho), \quad 0 \leqslant \rho \leqslant 1,
$$




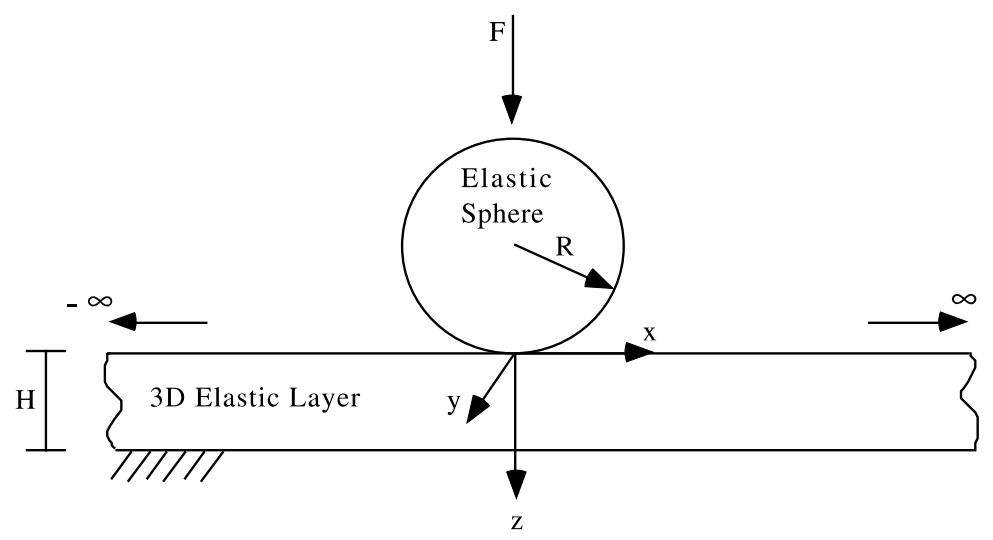

Fig. 2. Schematic diagram for contact between a sphere and an elastic layer.

$$
\int_{0}^{\infty} Q(s) J_{0}(s \rho) s \mathrm{~d} s=0, \quad \rho>1,
$$

where

$$
Q(s)=\int_{0}^{\infty} q(\rho) J_{0}(s \rho) \mathrm{d} \rho,
$$

$J_{0}(s \rho)$ represents the Bessel function of the zeroth kind, $s$ is a transform variable, and $\rho$ is a nondimensional radius, $r / a$, where $a$ is defined as the radius of the contact area, initially unknown prior to solution.

Here the function, $\varphi(\rho)$, represents the shape of the indenter, $\delta$ is the maximum relative approach, and $q(\rho)$ is the nondimensionalized contacting pressure on the indenter.

$$
\begin{aligned}
Q(\alpha)= & \frac{2}{\pi} \frac{\delta}{c_{1}+c_{2}} \frac{\sin \alpha}{\alpha}-\frac{2}{\pi} \frac{1}{c_{1}+c_{2}}\left[\cos \alpha \int_{0}^{1} \frac{y \varphi(y)}{\sqrt{1-y^{2}}} \mathrm{~d} y+\int_{0}^{1} \int_{0}^{1} \frac{y \varphi(y) \alpha u \sin (\alpha u)}{\sqrt{1-y^{2}}} \mathrm{~d} y \mathrm{~d} u\right] \\
& +\frac{2}{\pi} \frac{c_{1}}{c_{1}+c_{2}} \int_{0}^{\infty} \int_{0}^{1} \cos (\alpha u) \cos (\gamma u) B(2 \gamma h) Q(\gamma) \mathrm{d} \gamma \mathrm{d} u,
\end{aligned}
$$

where

$$
B(2 \gamma h)=\frac{1+2 \gamma h-\mathrm{e}^{-2 \gamma h}}{2 \gamma h+\sinh (2 \gamma h)}, \quad c_{1}=\frac{1-v_{1}}{G_{1}}, \quad \text { and } \quad c_{2}=\frac{1-v_{2}}{G_{2}} .
$$

$G_{1}$ and $v_{1}$ are shear modulus and Poisson's ratio of the indenter, and $G_{2}$ and $v_{2}$ are those for the layer, which represents the cushion. Keer also shows that the difference in the formulation to solve the contact problem of rigid spherical indenter and an elastic layer with finite thickness is $c_{2}=0$ in Eq. (13). In that case, the governing integral equation is the same as that derived by Vorovich and Ustinov, 1959. After manipulating the integral equation, we can find the contact radius by Eq. (14), the nondimensional contact stress by Eq. (15), the peak pressure by Eq. (16), and the maximum relative approach by Eq. (17).

$$
\begin{aligned}
& F=a_{(i)} \int_{0}^{2 \pi} \int_{0}^{q} q_{(i)}(\rho) \rho \mathrm{d} \rho \mathrm{d} \theta, \\
& q_{(i)}(\rho)=\int_{0}^{\infty} Q_{(i)}(\gamma) J_{0}(\gamma \rho) \gamma \mathrm{d} \gamma,
\end{aligned}
$$




$$
\begin{aligned}
& P_{(i) \max }=\frac{1}{a_{(i)}} \int_{0}^{\infty} Q_{(i)}(\gamma) \gamma \mathrm{d} \gamma, \\
& \delta_{(i)}=\int_{0}^{1} \frac{\varphi(y) y+\varphi^{\prime}(y) y^{2}}{\sqrt{1-y^{2}}} \mathrm{~d} y-c_{1} \int_{0}^{\infty} B(2 \gamma h) Q_{(i)}(\gamma) \cos \gamma \mathrm{d} \gamma,
\end{aligned}
$$

where $i=0,1,2,3, \ldots, k$, corresponding to each asymptotic order. $Q_{(i)}(\alpha)$ can be computed as follows.

$$
\begin{aligned}
& Q_{(0)}(\alpha)=\frac{2}{\pi} \frac{1}{c_{1}+c_{2}} \int_{0}^{1} \int_{0}^{1} \frac{\sin (\alpha u)}{\alpha} \frac{2 y^{2} \varphi^{\prime}(y u)+u y^{3} \varphi^{\prime \prime}(y u)}{\sqrt{1-y^{2}}} \mathrm{~d} y \mathrm{~d} u \\
& Q_{(i)}(\alpha)=Q_{(0)}(\alpha)+\frac{2}{\pi} \frac{1}{c_{1}+c_{2}} \frac{1}{\alpha} \int_{0}^{\infty} \int_{0}^{1} \gamma B(2 \gamma h) Q_{(i)}(\gamma) \sin (\alpha u) \sin (\gamma u) \mathrm{d} \gamma \mathrm{d} u
\end{aligned}
$$

To simplify the mathematical manipulation, if we assume the shape of the indenter is three-dimensional parabolic, Eqs. (14) and (15) can be integrated analytically and then the governing integral equation can be solved analytically as follows at each asymptotic order, based on the Neuman series expansion technique (Keer, 1964).

At the zero-order,

$$
\begin{aligned}
& F_{(0)}=\frac{8 a^{3}}{3 R} \frac{1}{c_{1}+c_{2}}, \\
& q_{(0)}(\rho)=\frac{4 a^{2}}{\pi R} \frac{1}{c_{1}+c_{2}} \sqrt{1-\rho^{2}}, \\
& P_{(0) \max }=q_{(0)}(0) / a_{(0)} .
\end{aligned}
$$

At the first-order,

$$
\begin{aligned}
& F_{(1)}=\frac{8 a^{3}}{3 R} \frac{1}{c_{1}+c_{2}}\left[1+\left(\frac{c_{1}}{c_{1}+c_{2}}\right)\left(\frac{0.337}{h^{3}}-\frac{0.342}{h^{5}}+\frac{0.037}{h^{7}}-\cdots\right)\right], \\
& \begin{aligned}
q_{(1)}(\rho)= & \frac{4 a^{2}}{\pi R} \frac{1}{c_{1}+c_{2}} \sqrt{1-p^{2}}\left\{1+\left(\frac{c_{1}}{c_{1}+c_{2}}\right)\left[\left(\frac{0.337}{h^{3}}-\frac{0.266}{h^{5}}+\frac{0.024}{h^{7}}-\cdots\right) \rho^{2}\right.\right. \\
& \left.\left.\quad+\left(-\frac{0.19}{h^{5}}+\frac{0.029}{h^{7}}+\cdots\right)+\rho^{4} \frac{0.01}{h^{7}}\right]\right\},
\end{aligned} \\
& P_{(1) \max }=q_{(1)}(0) / a_{(1)} .
\end{aligned}
$$

At the second-order,

$$
\begin{aligned}
& F_{(2)}=F_{(1)}+\frac{8 a^{3}}{3 R} \frac{1}{c_{1}+c_{2}}\left(\frac{c_{1}}{c_{1}+c_{2}}\right)^{2}\left(\frac{0.114}{h^{6}}-\frac{0.23}{h^{8}}\right), \\
& q_{(2)}=q_{(1)}+\sqrt{1+\rho^{2}} \frac{4 a^{2}}{\pi R} \frac{1}{c_{1}+c_{2}}\left(\frac{c_{1}}{c_{1}+c_{2}}\right)^{2}\left(\frac{0.114}{h^{6}}-\frac{0.205}{h^{8}}-\rho^{2} \frac{0.064}{h^{8}}+\cdots\right), \\
& P_{(2) \max }=q_{(2)}(0) / a_{(2)} .
\end{aligned}
$$


The Neuman series solutions, Eqs. (20)-(28), are similar to those in the paper (Keer, 1964). It was observed that Keer's results did not converge appropriately to the Hertz solution. The analysis was repeated and several errors were found in Keer's results. These errors are corrected in the present analysis.

\section{Results and discussion}

The numerical calculation of investigating the influences of each parameter in the Hertz theory on the contact of two spheres was performed. First, to illustrate the general characteristic of the contact pressure distribution, Fig. 3 shows the pressure profile along the radius on the contact surface, results of Eq. (1). The Young's modulus and Poisson's ratio of the spherical indenter are assumed as $1 \mathrm{MPa}$ and 0.49 respectively. These figures approximate the material properties of human soft tissue. The Poisson's ratio of the cushion in this example is set equal to 0.32 , which represents the Poisson's ratio of conventional polymer foams. The radius of the indenter is $0.15 \mathrm{~m}$ to model typical human buttocks, and the shape of the cushion is assumed to be perfectly flat. The external loading is $784 \mathrm{~N}$, which represents the weight of a $80-\mathrm{kg}$ person. For stiffer cushions the pressure will concentrate at the center of the contact region, and have a smaller contact region. However, the pressure will be smoothed out and lowered in magnitude for softer cushions, and, as we can envisage, the deformation will increase simultaneously. For sufficiently soft cushions, the Hertz theory loses validity since the contact region is no longer small compared to the indenter radius. Based on the theory of elasticity, this pressure profile is independent of the cushion's Poisson's ratio. However, the following investigations will show that the negative Poisson's ratio cushions reduce peak contact pressure.

Fig. 4 shows the relationship between the maximum pressure, maximum relative approach, and the ratio of the Young's modulus of the two contacting bodies, on a log-log scale. Following Eq. (8), the trend of the pressure curve (open circle symbols) shows that reducing the cushion's Young's modulus can largely reduce the maximum pressure on the indenter. However, the maximum relative motion will greatly increase when the ratio of the two moduli is sufficiently small. Although the Hertz theory, since it assumes linear elasticity and spherical or semi-infinite regions, cannot predict whether the cushion has bottomed out, a maximum indentation or approach greater than $0.1 \mathrm{~m}$ exceeds the thickness of most real cushions and therefore

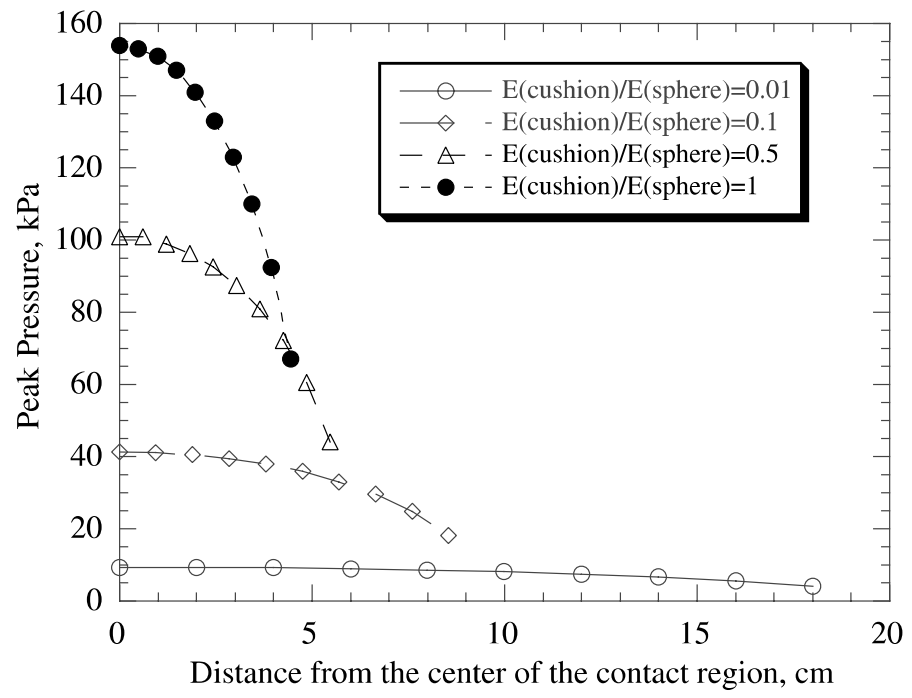

Fig. 3. Pressure profile in the contact region between an elastic sphere and half-space cushion, Eq. (1). 


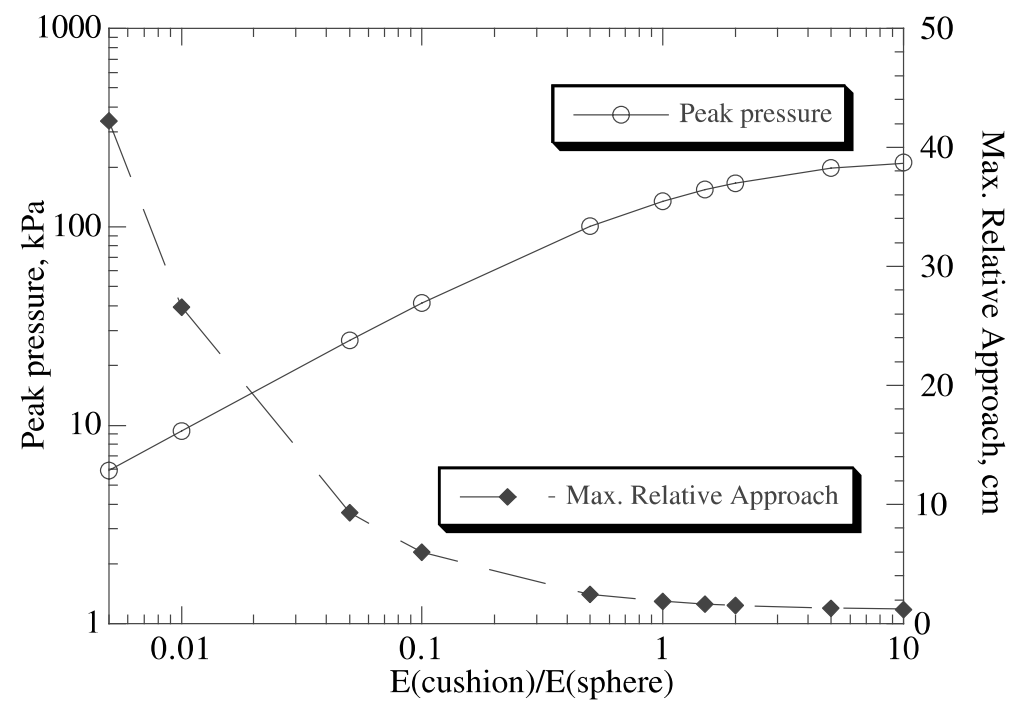

Fig. 4. Peak pressure and maximum relative motion vs. Young's modulus ratio, Eqs. (3) and (8).

indicates the cushion stiffness is so small that bottoming would occur in practice. In that situation, the peak pressure would be much greater than that predicted here. Later a more sophisticated analysis model will clearly support this argument.

The relationship, calculated from Eqs. (8) and (9), of maximum pressure, maximum relative approach, and the cushion's Poisson's ratio to different Young's modulus ratios $\left(E_{\mathrm{c}} / E_{\mathrm{s}}\right)$ is shown in Fig. 5. The open symbols represent the maximum pressure, and solid symbols are maximum relative motion. As the Poisson's ratio decreases from 0.5 to -1 with holding the Young's modulus ratio and all other geometrical and loading parameters, the minimum of peak pressure will occur at the Poisson's ratio of zero. At this point, the maximum relative motion reaches its maximum in the Poisson's ratio range of isotropic materials. In addition, the maximum of the peak pressure will occur at the Poisson's ratio of -1 . It can be understood that the trend of the curves with open symbols (diamond, square, and circle) show that the peak pressure on the indenter decreases as the Young's modulus of the cushion decreases, i.e. ratio of $E_{\mathrm{s}} / E_{\mathrm{c}}$ increases.

Figs. 6 and 7 show the relationship between the peak pressure, shear modulus, and Poisson's ratio of the cushion at different shear modulus ratios. From these figures, it can be seen that, based on the Hertz solutions, reducing cushion shear moduli can largely decrease the peak pressure, and reducing the Poisson's ratio also can diminish pressure but to a lesser extent. The same equations are used, Eqs. (8) and (9), but the constant $D$ was computed from Eq. (4), because, in this case, the shear moduli are assumed to be known. The shear modulus and Poisson's ratio of the indenter are assumed as $0.33 \mathrm{MPa}$ and 0.49 , respectively. The geometrical and loading conditions are the same as previous example. The open symbols represent the maximum pressure, and solid symbols are maximum relative motion. When the shear modulus of the cushion is much larger than that of the indenter, Poisson's ratio will have no influence both on the magnitude of maximum pressure and maximum relative motion, which satisfies our physical intuition. However, the general trends of the open-symbol curves show maximum pressure decreases with the decrease of the Poisson's ratio. The minimum of the peak pressure occurs when the Poisson's ratio of the cushion reaches -1 . When the difference of shear modulus between cushion and indenter is larger, this kind of decreases is more apparent. On the other hand, the trends of the solid-symbol curves show the maximum relative motion increases with the decrease of the Poisson's ratio. Observing the trend of the curves at some fixed Poisson's ratio, the maximum pressure decreases when the cushion becomes softer on shear resistance. 


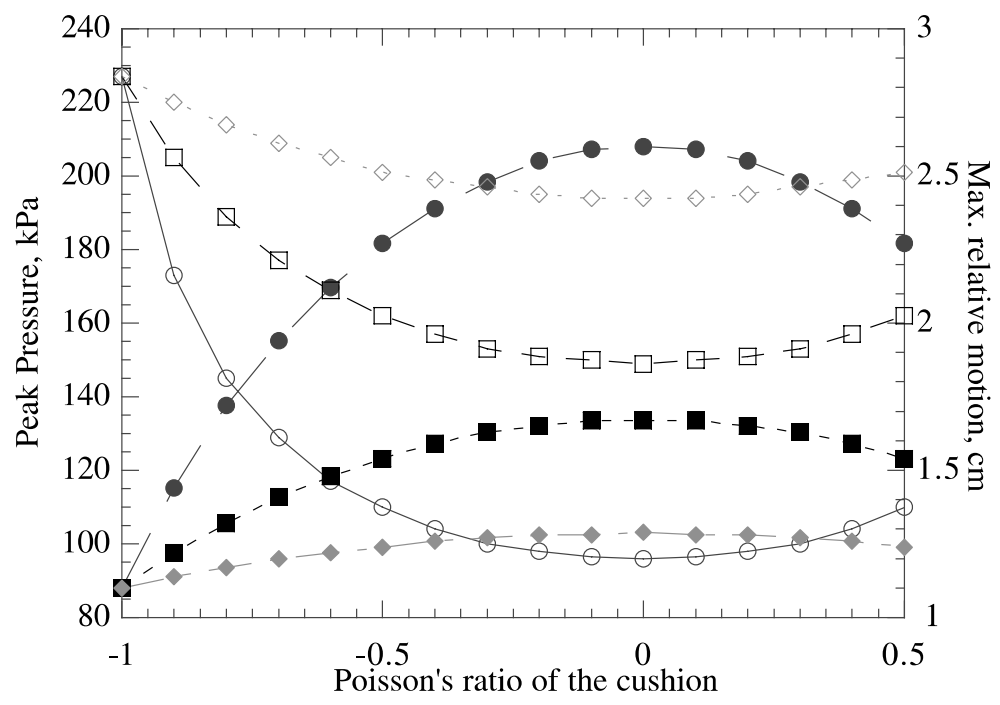

$$
\begin{gathered}
-\bigcirc \text { - Max. Pres., E(cushion) } / \mathrm{E}(\text { sphere })=0.5 \\
-\square-\text { Max. Pres., E(cushion) } / \mathrm{E}(\text { sphere })=1.5 \\
-\Leftrightarrow-\text { Max. Pres., E(cushion) } / \mathrm{E}(\text { sphere })=5
\end{gathered}
$$

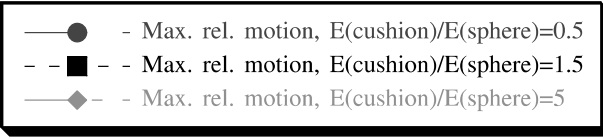

Fig. 5. Peak pressure and maximum relative motion vs. Poisson's ratio of the cushion at different Young's modulus ratios, Eqs. (3), (8) and (9).

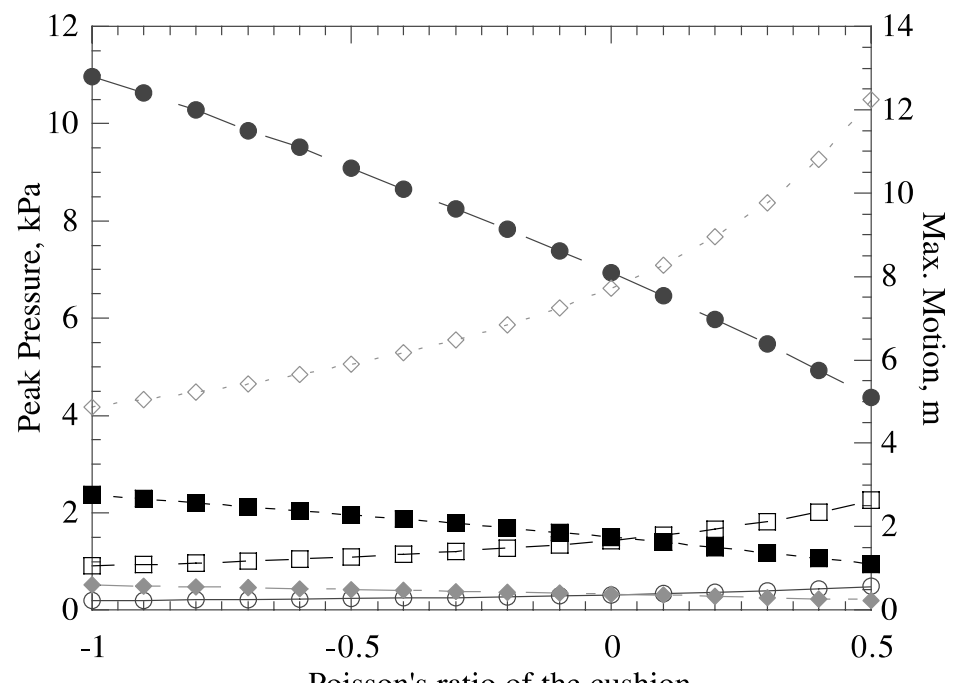

Poisson's ratio of the cushion

- Max. Pressure, G(cushion)/G(sphere) $=0.0001$

$-\square$ - Max. Pressure, G(cushion)/G(sphere) $=0.001$

Max. Pressure, G(cushion) $/ \mathrm{G}($ sphere $)=0.01$
- Max. Motion, G(cushion) $/ \mathrm{G}($ sphere $)=0.0001$

- - - Max. Motion, G(cushion)/G(sphere $)=0.001$

- Max. Motion, G(cushion) $/ \mathrm{G}($ sphere $)=0.01$

Fig. 6. Peak pressure and maximum motion vs. Poisson's ratio of the cushion at different shear modulus ratios $(0.0001,0.001,0.01)$, Eqs. (4), (8) and (9). 

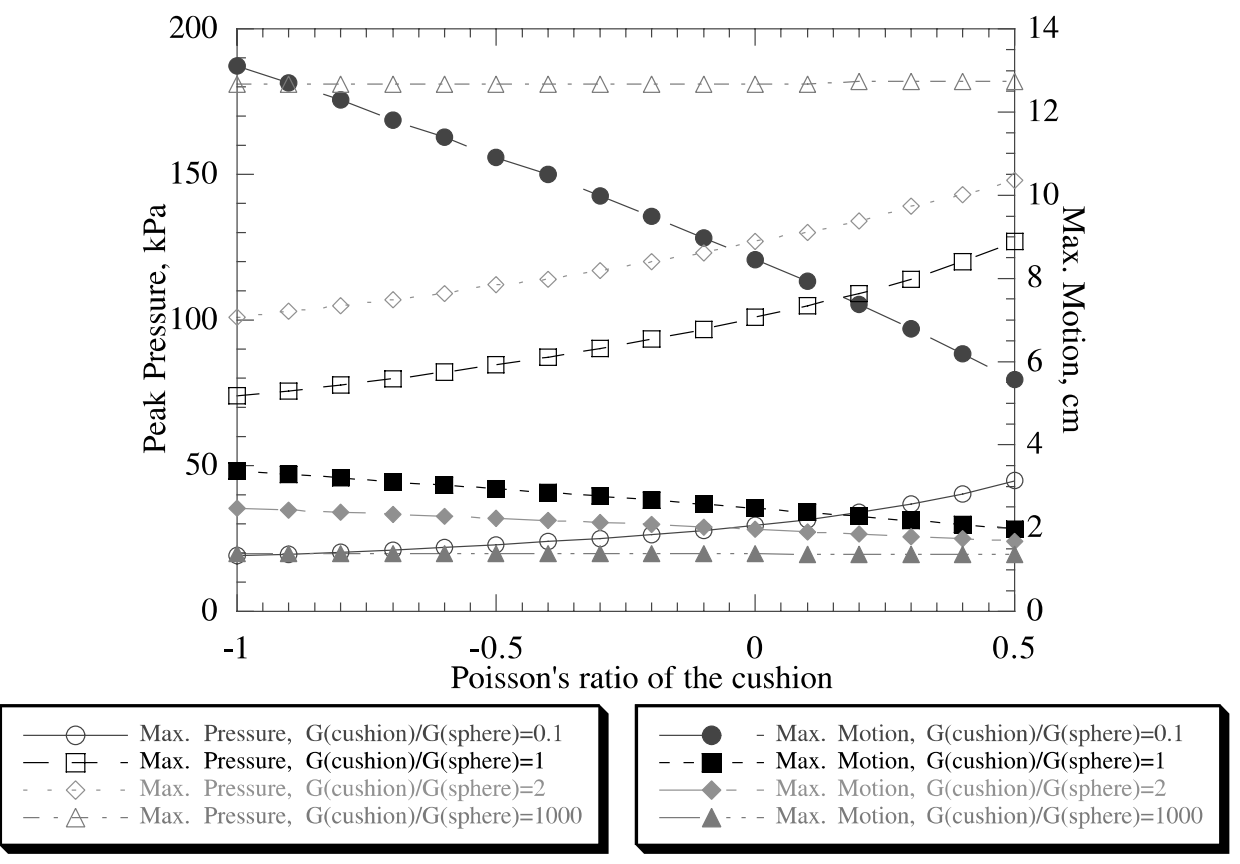

Fig. 7. Peak pressure and maximum motion vs. Poisson's ratio of the cushion at different shear modulus ratios $(0.1,1,2,1000)$, Eqs. (4), (8) and (9).

Again, cases of indentation (approach) greater than $0.1 \mathrm{~m}$, notably in Fig. 4 are not realistic in terms of cushions. Cushions made of such a soft material would bottom out in practice.

In the analytical model of 3D elasticity with finite thickness, by using the truncated series, Eqs. (14)-(19), to represent their original integration, Eqs. (20)-(28), at each asymptotic level, Fig. 8 shows the relationship between peak contact pressure, maximum relative approach and the thickness of the cushion. It is noteworthy that the coincidence of the Hertz solution and the solution from zero-order asymptotic expansion indicates the physical meaning of the zero-order solution in this asymptotic formulation. Although the explicit physical meaning for higher order asymptotic terms is not so clear, the more terms we use, the more the contribution from the finite thickness will affect the results. Fixing all geometrical and material parameters, and varying the thickness of the cushion only, we observe the peak pressure increases as the thickness decreases, which satisfies one's physical expectation of the effect of bottoming out. However, the distance of relative approach decreases with the thickness. Due to the solution including the errors from the second-order approximation in the asymptotic expansion and the truncated series in integration, the prediction of pressure and motion is only reliable for a moderately thin cushion. In this typical case, the percentage of deviations of the peak pressure due to the thickness is $5.3 \%$ for first-order and $13.2 \%$ for second-order solution for a $0.06 \mathrm{~m}$-thick cushion. For a $0.1 \mathrm{~m}$-thick cushion, the difference is less than $1 \%$. Based on the general convergence pattern for asymptotic solutions, the exact solution for peak pressure can be found between the first- and second-order solution. The thickness influence on the relative approach is $3.7 \%$ for $0.1 \mathrm{~m}$-thick cushion and $7 \%$ for $0.06 \mathrm{~m}$-thick cushion. In the calculation, the material properties are as follows. For the indenter, they are $0.33 \mathrm{MPa}$ for shear modulus, and 0.49 for Poisson's ratio. For the cushion, the shear modulus and Poisson's ratio are $0.033 \mathrm{MPa}$ and 0.32 . The radius of the indenter is $0.15 \mathrm{~m}$. 


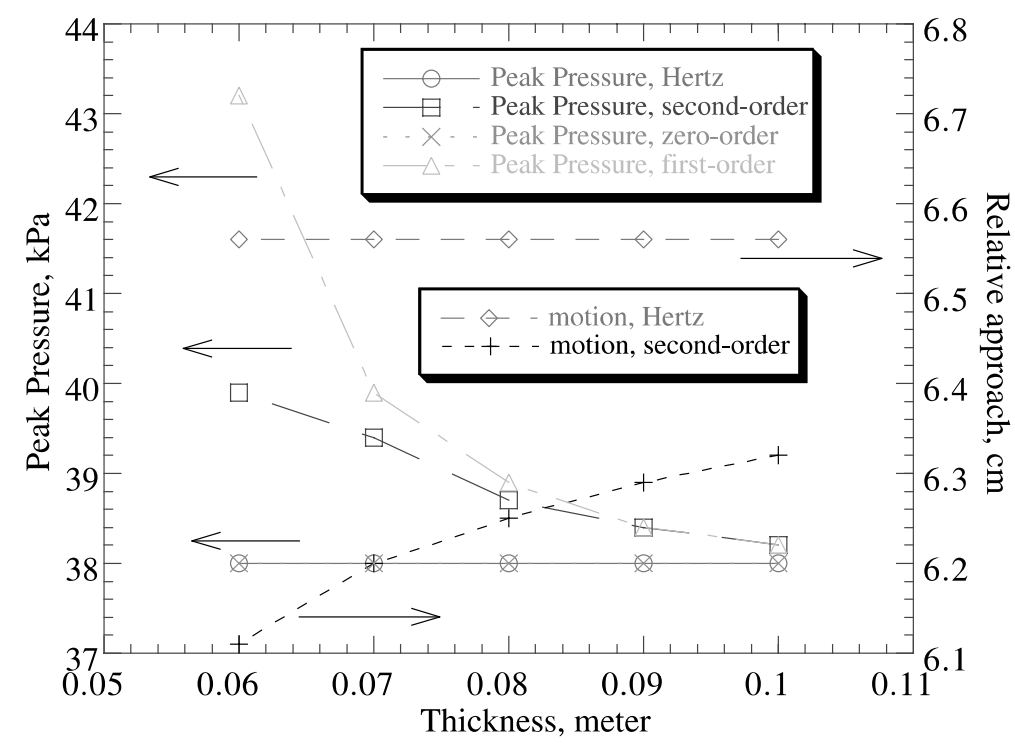

Fig. 8. Peak pressure and maximum relative approach vs. thickness, finite thickness model, Eqs. (14)-(19).

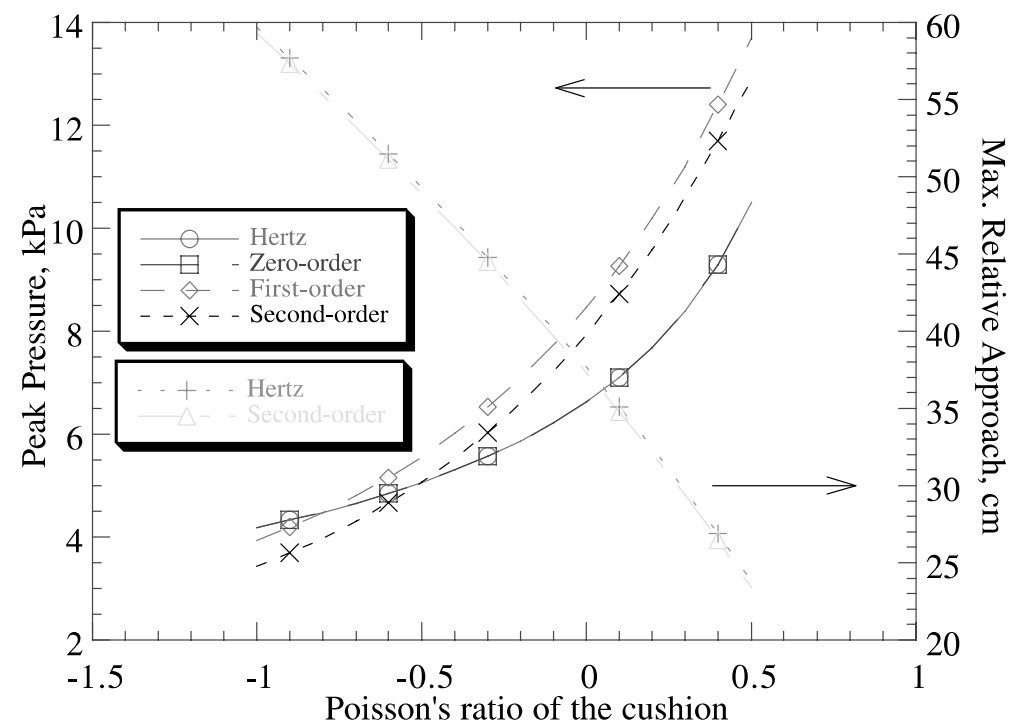

Fig. 9. Peak pressure and maximum relative approach vs. Poisson's ratio of the cushion at $G($ indenter $) / G($ cushion $)=100$, finitethickness model, Eqs. (14)-(19).

In contrast to Hertz theory, the predictions made by the finite-thickness model are plotted as Figs. 9 and 10 to show the influence of cushions' Poisson's ratio at the shear moduli ratios of 100 and 10, respectively. The thickness of the cushion is chosen to be $0.07 \mathrm{~m}$, and the shear modulus and Poisson's ratio of the indenter are $0.33 \mathrm{MPa}$ and 0.49 . The radius of the indenter is $0.15 \mathrm{~m}$. The modulus ratio 100 corresponds to a very soft cushion, $E=3.3 \mathrm{kPa}$; a ratio 10 corresponds to a more realistic cushion, $E=33 \mathrm{kPa}$. Although the second-order asymptotic solutions are suitable only for moderate soft cushions, both in the Hertz 


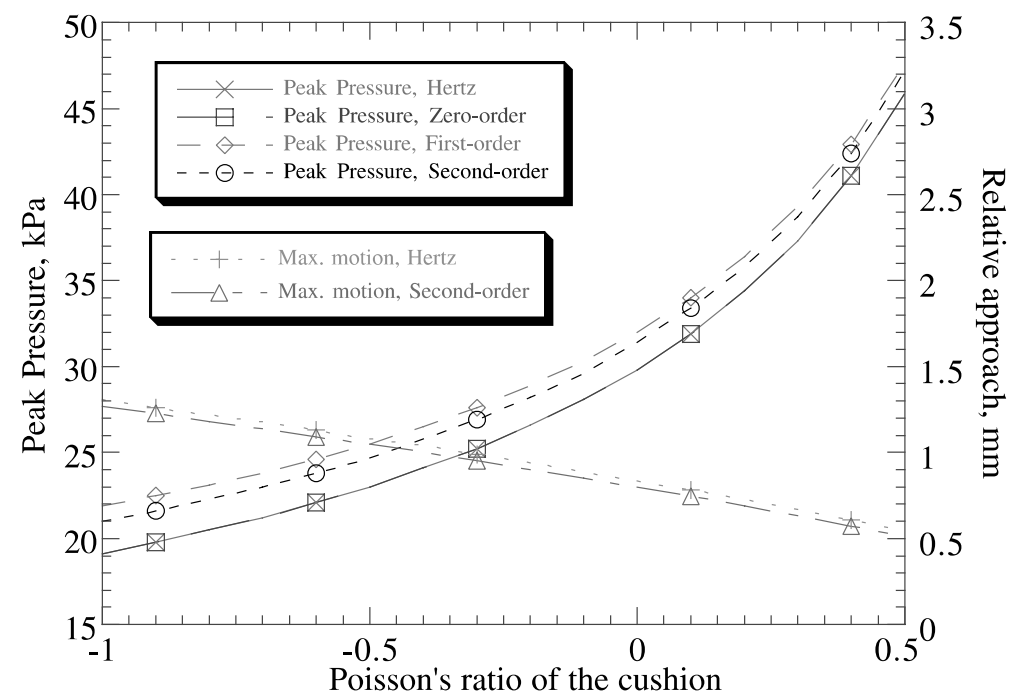

Fig. 10. Peak pressure and maximum relative approach vs. Poisson's ratio of the cushion at $\mathrm{G}($ indenter $) / \mathrm{G}(\mathrm{cushion})=10$, finitethickness model, Eqs. (14)-(19).

solution and in the finite-thickness second-order analysis, a reduction in cushion Poisson's ratio, including negative Poisson's ratios, gives rise to a reduction in peak pressure.

The results of varying the curvature of the cushion are shown in Fig. 11, following Eqs. (1) and (2). The assumed Young's modulus and Poisson's ratio of the indenter are $1 \mathrm{MPa}$ and 0.49 respectively, and those of the cushion are $0.2 \mathrm{MPa}$ and 0.32 . The radius of the indenter is held to be $0.15 \mathrm{~m}$, and the external load is $784 \mathrm{~N}$. It is observed that when the indenter has smaller curvature, that is a larger radius of curvature; it is

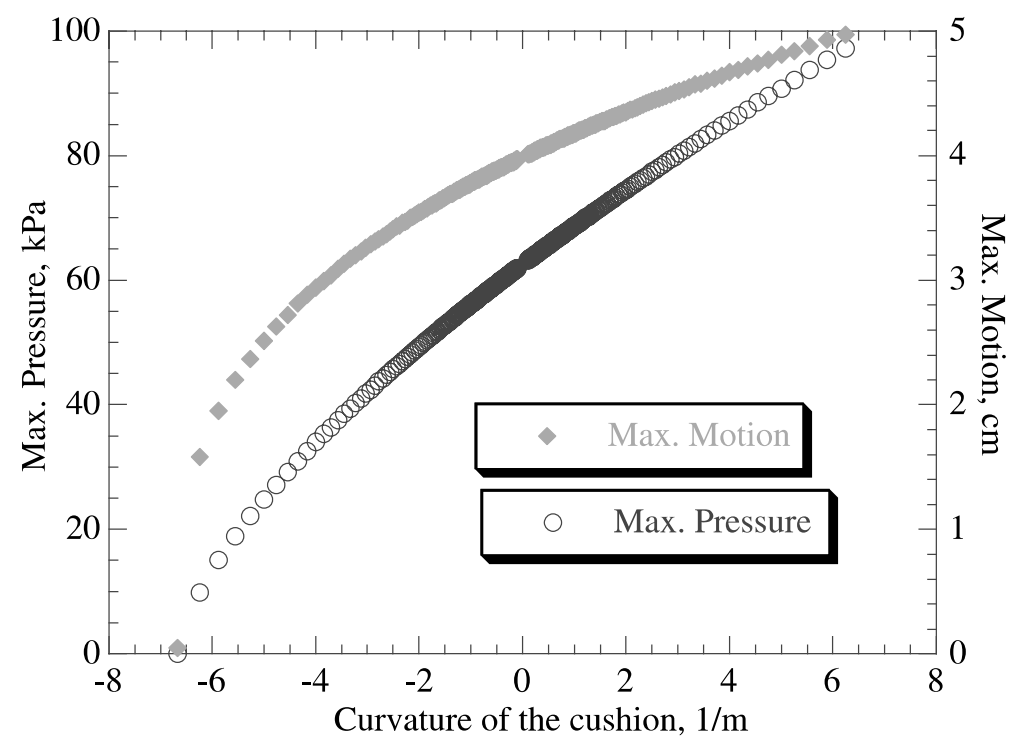

Fig. 11. Maximum pressure and maximum motion vs. the curvature of the cushion. Negative curvature corresponds to a contoured cushion, shaped to fit the shape of the spherical indenter, Eqs. (1) and (2). 
flatter, the peak pressure on it is also smaller. However, if the indenter represents human buttocks, any change in their size will likely be accompanied by a different in body weight.

Through adjusting the shape of the cushion, it is found from Eqs. (1) and (2) that when the cushion has negative curvature, i.e. spherical-cavity or concave shape, both the maximum pressure and relative motion will be largely reduced. Furthermore, the contact pressure becomes zero when the shapes of the indenter and spherical-cavity cushion are conformed. This indicates the inaccuracy of the Hertz model for this application because of the violation of model assumptions. Specifically, in a contoured cushion, the area of contact is no longer ever approximately smaller than the radius of curvature of the indenter (buttocks). This problem might be solved by using a modified theory (Goodman and Keer, 1965). However, their solutions are restricted to materials with Poisson's ratio of 0.25 .

\section{Conclusions}

Based on the current analytical contact analysis models, a soft cushion helps reduce pressure. However, a cushion which is too soft will bottom out, resulting in increased pressure. The $3 \mathrm{D}$ elasticity finite thickness solution used here incorporates some aspects of bottoming. However a full understanding of bottoming is limited by the complexity of the asymptotic solutions and by the fact the assumption of linearity excludes the material nonlinearity of the foam and human tissue at high strain.

The results obtained from the models suggest that a negative Poisson's ratio cushion reduces the peak pressure if the cushion shear modulus is held constant as Poisson's ratio is varied. If the cushion Young's modulus is held constant, the optimal Poisson's ratio is zero. This conclusion is robust and consistent with respect to both the analytical models.

A contoured cushion substantially reduces peak pressure. Combining the concepts of contoured shape and negative Poisson's ratio materials offers superior cushion performance.

The analysis allows the geometrical nonlinearity of contact but assumes material linearity. The nonlinearity of foam and tissue at large strain is acknowledged. The results from the present analysis are exact only for linear material behavior. Although neither human tissue nor conventional foam cushions are perfect linear elastic materials, the negative Poisson's ratio foam can behave linearly in compression up to $40 \%$ strain. The insight gained in the present analysis may be of use in designing new cushions, and also as a guide for future analysis which incorporates more of the complexities of the problem. Such studies may incorporate analytical treatment of material nonlinearity and nonaffine deformation, or finite element methods.

\section{References}

Brienza, D.M., Karg, P.E., Brubaker, C.E., 1996. Seat cushion design for elderly wheelchair users based on minimization of soft tissue deformation using stiffness and pressure measurements. IEEE Trans. Rehab. Eng. 4 (4), 320-327.

Brienza, D.M., Lin, C.T., Karg, P.E., 1999. A method for custom-contoured cushion design using interface pressure measurements. IEEE Trans. Rehab. Eng. 7 (1), 99-108.

Choi, J.B., Lakes, R.S., 1992. Nonlinear properties of polymer cellular materials with a negative Poisson's ratio. J. Mater. Sci. 27, 4678-4684.

Dabnichki, P.A., Crocombe, A.D., Hughes, S.C., 1994. Deformation and stress analysis of supported buttock contact, Proceedings of the Institution of Mechanical Engineers. J. Eng. Med.-Part H 208 (1), 9-17.

Fessler, H., Ollerton, E., 1957. Contact stresses in toroids under radial loads. British J. Appl. Phys. 8.

Goodman, L.E., Keer, L.M., 1965. The contact stress problem for an elastic sphere indenting an elastic cavity. Int. J. Solids Struct. 1, 407-415.

Johnson, K.L., 1985. Contact Mechanics. Cambridge University Press, Cambridge, London. 
Kalker, J.J., van Ranfen, Y., 1972. A minimum principle for frictionless elastic contact with application to non-Hertzian half-space contact problems. J. Eng. Math. 6, 193.

Keer, L.M., 1964. The contact stress problem for an elastic sphere indenting an elastic layer. J. App. Mech., $143-145$.

Kosior, F., Guyot, N., Maurice, G., 1999. Analysis of frictional contact problem using boundary element method and domain decomposition method. Int. J. Numer. Meth. Eng. 46, 65-82.

Lakes, R.S., 1987. Foam structures with a negative Poisson's ratio. Science 235, 1038-1040.

Lakes, R.S., 1992. Saint Venant end effects for materials with negative Poisson's ratios. J. Appl. Mech. 59, 744-746.

Lakes, R.S., 1993. Design considerations for materials with negative Poisson's ratios. Trans. ASME J. Mech. Des. 115, 696-700.

Loureiro, M.A., Lakes, R.S., 1997. Scale-up of transformation of negative Poisson's ratio foam: Slabs. Cellular Polym. 16, $349-363$.

Lowe, A., Lakes, R.S., 2000. Negative Poisson's ratio foam as seat cushion material. Cellular Polym. 19 (3), $157-167$.

Paul, B., Hashemi, J., 1980. Contact pressures on closely conforming elastic bodies. In: Sym. on Solid Contact and Lubrication, AMD39. ASME, New York.

Sakamoto, M., Li, G., Hara, T., Chao, Y.S., 1996. A new method for theoretical analysis of static indentation test. J. Biomech. 29 (5), 679-685.

Shen, W., Parsons, K.C., 1997. Validity and reliability of rating scales for seated pressure discomfort. Int. J. Ind.1 Ergonom. 20, 441461.

Singh, K.P., Paul, B., 1974. Numerical solution of non-Hertzian elastic contact problems. J. Appl. Mech., 484-490.

Smith, D.M., 1995. Pressure ulcers in the nursing home, [review]. Annals Int. Med. 123 (6), 433-442.

Todd, A.B., Thacker, G.J., 1994. Three-dimensional computer model of the human buttocks in vivo. J. Rehab. Res. Develop. 31 (2), 111-119.

Vorovich, I.I., Ustinov, Iu.A., 1959. Pressure of a die on an elastic layer of finite thickness. J. Appl. Math. Mech. 23, 637-650.

Yoffe, E.H., 1984. Modified Hertz theory for spherical indentation. Philos. Mag. A 50 (6), 813-828. 